\title{
EDUCAÇÃO, SAÚDE E AMBIENTE: AS CONCEPÇÕES DOS AGENTES COMUNITÁRIOS DE SAÚDE
}

\author{
EDUCATION, HEALTH AND ENVIRONMENT: THE VISION OF \\ COMMUNITY HEALTH STAFF \\ Francisco Tavares Guimarães ${ }^{1}$, Rodrigo Roger Vitorino ${ }^{1}$, Pedro Henrique Netto Cezar ${ }^{1}$, \\ Joana Ferrez de Castro ${ }^{2}$, Rosângela Mitre Minardi Cotta ${ }^{3}$, José Roberto Bittencourt \\ Costa $^{1}$ e Rodrigo Siqueira-Batista ${ }^{3}$ \\ ${ }^{1}$ Centro Universitário Serra dos Órgãos - UNIFESO \\ ${ }^{2}$ Universidade Presidente Antônio Carlos - UNIPAC \\ ${ }^{3}$ Universidade Federal de Viçosa - UFV
}

\section{RESUMO}

O presente artigo tem por escopo analisar as concepções dos agentes comunitários de saúde (ACS), de quatro unidades da Estratégia de Saúde da Família (ESF) do município de Teresópolis-RJ, acerca da relação entre o ambiente e o processo saúde-doença, à luz dos referenciais da educação ambiental (EA). Para tanto, foi realizado um estudo qualitativo, a partir do conteúdo das respostas obtidas por meio de entrevista. Pôde-se observar que as percepções dos ACS são consistentes, no sentido de correlacionar a existência de um ambiente degradado com o processo saúde-doença. Entretanto, evidenciaram-se dificuldades, do ponto de vista dos ACS, em relação (1) ao trabalho desta idéias junto à comunidade e (2) à inoperância do poder público em viabilizar processos de articulação saúde-ambiente, aspectos essenciais para promoção da saúde (PS) e o cultivo de ambientes saudáveis.

Palavras-chave: Atenção primária à saúde; Educação ambiental; Saúde da família.

\begin{abstract}
This paper aims at examining the conceptions of community health agents (CHA), four units of the Family Health Strategy (FHS) in Teresópolis-RJ, on the relationship between the environment and the health-disease, light of the benchmarks of environmental education (EE). For this, a qualitative study was carried out from the content of the responses obtained through interviews. It was observed that perceptions of the ACS are consistent, in order to correlate the existence of a degraded environment with the health-illness. However, difficulties have become apparent, from the standpoint of CHA in relation (1) to work with the community of ideas and (2) the ineffectiveness of government in enabling processes of health-environment links, which are essential for health promotion (HP) and the cultivation of healthy environments.
\end{abstract}

Key-words: Environmental education; Family health; Primary health care. 


\section{INTRODUÇÃO}

A vinculação entre o meio ambiente e o processo de adoecimento é amplamente reconhecida na história da medicina ocidental (AZEVEDO, 2003; HELLER, 1997; MONKEN, et al., 2008; PAULA, 1997; SAVASTANO, 1980; SRINIVASAN, et al., 2003). De fato, Hipócrates, no livro Dos Ares, da água e dos Lugares, já destacava o papel do reconhecimento dos fatores ambientais para a prática clínica, pontuando ser indispensável ao bom médico conhecer o território e as condições nas quais vive o seu paciente (HIPÓCRATES, 2002; SIQUEIRA-BATISTA, 2003):

Há observações que ajudam a prever como será o ano, se salubre ou insalubre. Se não houver nenhuma alteração nos sinais que acompanham o poente e o levante dos astros, se as chuvas caírem no outono, se o inverno for moderado, nem muito ameno nem muito frio, se durante a primavera e o verão as chuvas seguirem a ordem dessas duas estações, naturalmente o ano será muito saudável. Ao contrário, quando a um inverno seco e boreal suceder-se uma primavera chuvosa e austral, o verão trará necessariamente febres, oftalmias e disenterias. [...] Se o inverno for austral chuvoso e ameno, e a primavera boreal, seca e fria, as mulheres que estiverem grávidas e que deverão dar à luz na primavera abortarão, e aquelas que chegarem ao termo, trarão ao mundo crianças fracas e doentias que morrerão imediatamente ou que viverão fracas e doentes. Tais são os acidentes próprios das mulheres. $\mathrm{O}$ restante da população estará sujeita às disenterias e às oftalmias secas; algumas pessoas apresentarão defluxões na cabeça e no pulmão (HIPÓCRATES, 2002, p. 99-101)

A atualidade do trabalho do mestre de Cós é atestada pelo destaque que vem sendo dado, nas últimas décadas, às relações saúde-ambiente:

A saúde dos seres humanos é predominantemente determinada, não por intervenção médica, mas pelo comportamento, pela alimentação e pela natureza de seu meio ambiente. Como essas variáveis diferem de cultura para cultura, cada uma tem suas próprias enfermidades características, e, na medida em que mudam gradualmente a alimentação, o comportamento e as situações ambientais, mudam também os tipos de doença (CAPRA, 2006; p. 131)

A compreensão destes elementos impõe a revisão das práticas de saúde, a partir da contextualização do fenômeno adoecimento ao espaço/tempo da existência do sujeito (GUIMARÃES, 2000); abre-se, com efeito, real possibilidade de atuação no âmbito da Estratégia de Saúde da Família (ESF) e do Programa de Agentes Comunitários de Saúde (PACS), os quais são modos para a reorientação da Atenção Primária à Saúde (APS) no Brasil, caracterizados, prioritariamente, por uma atuação territorial (BRASIL/DAB, 2013). 
A ESF foi criada em 1994 - inicialmente como PSF, Programa de Saúde da família -, como tentativa de reordenação do modelo de atenção à saúde, cabendo-lhe destaque no processo de consolidação do Sistema Único de Saúde (SUS) (BRONBERGER, 2003). As equipes da ESF são multidisciplinares, sendo constituídas por um médico, um enfermeiro, um auxiliar de enfermagem e de seis a oito agentes comunitários de saúde (ACS), os quais atuam em uma área adscrita com cerca de oitocentas famílias assistidas (aproximadamente 3500 pessoas). Esta proximidade ao sujeito residente na comunidade possibilita e estimula a formação de vínculos, elemento essencial para o adequado cuidado à saúde (CAMPOS, 2007; DUNCAN et al., 2006), alicerçado, especialmente, no trabalho do ACS. Este último representa uma peça fundamental na ESF, cabendo-lhe as seguintes habilidades e competências (BRASIL, 2003; GOMES, 2009):

(1) Identificar as condições do ambiente físico e social que constituem risco para a saúde de indivíduos e populações;

(2) Identificar a relação entre os problemas de saúde e as condições de vida com base nas interpretações obtidas;

(3) Estabelecer propostas de processos intersetoriais, visando o desenvolvimento do trabalho de promoção da saúde;

(4) Analisar os riscos sociais e ambientais à saúde, por microáreas de territorialização;

(5) Ter conhecimento das doenças mais comuns, por grupo etário, inserção social e distribuição geográfica, com ênfase nas características locais esperadas de atuação.

As prerrogativas do ACS acima descritas - estreitamente vinculadas às relações saúde-ambiente - pressupõem a adoção de uma decisiva ação de educador junto às comunidades, para o que a Educação Ambiental (EA) ganha substantiva relevância. Nestes termos, no capítulo 36 da Agenda 21, a EA é definida como o processo que busca:

[...] desenvolver uma população que seja consciente e preocupada com o meio ambiente e com os problemas que lhes são associados. Uma população que tenha conhecimentos, habilidades, atitudes, motivações e compromissos para trabalhar, individual e coletivamente, na busca de soluções para os problemas existentes e para a prevenção dos novos [...] (BRASIL, 2001, p. 438) 
Tendo essa premissa como referência, propõe-se que a EA seja um processo de formação - dinâmico e participativo -, no qual as pessoas envolvidas tornem-se agentes transformadores, buscando, ativamente, alternativas para a redução de impactos ambientais e para o controle social do uso dos recursos naturais. A incorporação da EA como atribuição do ACS poderá permitir que a comunidade assuma uma posição participativa diante das políticas ambientais (LOUREIRA, 2004), com profundo impacto no bojo da promoção da saúde (PS).

Com base nestas considerações, no presente estudo procurou analisar a percepção dos ACS de quatro unidades básicas de saúde da família (UBSF) do município de Teresópolis-RJ, acerca da relação entre o ambiente e o processo saúdedoença, à luz dos referenciais da EA.

\section{MÉTODOS}

\section{Área de estudo}

A investigação foi realizada na cidade de Teresópolis/RJ, a qual conta com cento e cinquenta mil e duzentos e sessenta e oito habitantes, perfazendo uma densidade demográfica de 195 habitantes $/ \mathrm{km}^{2}$ (IBGE, 2010). Está localizada a $93 \mathrm{~km}$ da capital do Estado e situada a 910 metros acima do nível do mar (IBGE, 2010).

Foram escolhidas quatro UBSF - de um total de 13 existentes no município -, duas localizadas na zona urbana (UBSF Rosário e UBSF Perpétuo) e duas na zona rural (UBSF Venda Nova e UBSF Vargem Grande) (Figura 1). Essa escolha teve por objetivo mapear possíveis diferenças em relação às interseções saúde-ambiente nos espaços urbano e rural.

\section{(INSERIR FIGURA 1)}

Figura 1. Unidades Básicas de Saúde da Família estudadas. Adaptado de www.telelistas.net.

\section{Procedimentos de coleta dos dados}

Participaram da pesquisa 21 Agentes Comunitários de Saúde - chamados doravante de ACS - vinculados às quatro equipes das referidas UBSF, os quais responderam perguntas abertas e fechadas durante procedimento de entrevista. As 
questões se destinavam à averiguação do nível de compreensão dos ACS acerca (1) do papel do meio ambiente como determinante do adoecimento e (2) do conceito da EA e de sua importância para a saúde (Quadro 1).

\section{(INSERIR QUADRO 1)}

Quadro 1. Perguntas do roteiro de entrevista aplicado aos agentes comunitários de saúde.

\section{Análise dos dados}

A apreciação dos dados foi estabelecida pelo método de análise de conteúdo, o qual, segundo Bardin (1977) diz respeito a um conjunto de técnicas de averiguação das comunicações, realizado a partir de procedimentos sistemáticos e objetivos de descrição do conteúdo das mensagens (BARDIN, 1977). Dentre as técnicas de análise de conteúdo optou-se pela análise temática (MINAYO, 2006). Assim, foram obedecidas as três etapas operacionais da análise temática, segundo as orientações de Minayo (2006): (1) Pré-análise, decomposta em leitura flutuante, constituição do corpus e formulação de hipóteses e objetivos a partir das respostas dos entrevistados; (2) Exploração do material; e (3) Tratamento dos resultados obtidos e interpretação.

\section{Aspectos éticos}

A pesquisa foi aprovada pelo Comitê de Ética em pesquisa do Instituto Federal de Educação, Ciência e Tecnologia do Rio de Janeiro (IFRJ) - Memorando de Aprovação no 008/09 -, em conformidade com o previsto na Resolução 196/96 do Conselho Nacional de Saúde e resoluções complementares. Os sujeitos de pesquisa, ACS, aceitaram, livremente, participar da investigação, mediante a assinatura do Termo de Consentimento Livre e Esclarecido (TCLE).

\section{RESULTADOS E DISCUSSÃO}

A partir da análise das respostas dos entrevistados foi possível criar categorias, cujos conteúdos manifestos e latentes apresentavam maior correlação.

\section{$O$ adoecimento das pessoas}


Para se analisar o conhecimento dos ACS acerca dos motivos que levam a população de suas comunidades adoecerem foi elaborada a primeira pergunta: " $O$ que faz as pessoas de sua comunidade adoecerem?".

Quanto às respostas dadas, fica claro o reconhecimento de diferentes fatores no processo de adoecimento. Particularmente, em relação ao ambiente, são destacados determinantes tanto na região urbana - falta de saneamento básico adequado e exposição de lixo a céu aberto - quanto na área rural - uso imprudente dos agrotóxicos. É evidente que intervenções no saneamento - capazes de minimizar o impacto negativo do meio ambiente sobre a saúde da população - está condicionada a transformações estruturais e institucionais (HELLER, 1997). De fato, observa-se que quanto maior a cobertura populacional por serviços adequados de esgotamento sanitário, em um país latino-americano ou caribenho, menor a mortalidade em menores de cinco anos de idade nestes países (TEIXEIRA \& PUNGIRIM, 2005).

\section{Relação do adoecimento com o ambiente}

Para verificar se o ACS correlaciona o fenômeno do adoecimento com o meio em que os membros da comunidade habitam, foi feita a pergunta "Você acredita que o ambiente em que as pessoas vivem pode levar ao aparecimento de doenças? Dê exemplo(s) dessa correlação”.

Fica evidente o conhecimento dos ACS no estabelecimento de correlações entre doença e ambiente insalubre. A enfermidade não é apenas originada da pobreza e da desigualdade, possuindo raízes no desequilíbrio ambiental - podendo-se citar, como exemplo, a moléstia de Chagas que é, antes de tudo, uma "doença ecológica" (GOMES et al., 2008; SIQUEIRA-BATISTA et al., 2011).

\section{Importância da capacitação em EA}

Com o intuito de apreciar a relevância atribuída pelo ACS à EA - e se estes foram instruídos para exercê-la - foi elaborada a pergunta: "Você já recebeu capacitação sobre meio ambiente ou educação ambiental? Você acha importante?". Dos entrevistados, somente quatro tiveram alguma capacitação sobre o tema, três na zona urbana e um na zona rural. Os demais, treze ACS, não referiram nenhum tipo de treinamento dessa natureza. 
Em relação ao conhecimento sobre o ambiente, os ACS foram unânimes em destacar a importância de dominar conceitos relativos à relação saúde-ambiente para o exercício de suas funções. A partir das respostas obtidas foi possível criar três categorias que expressam a finalidade de se conhecer os fundamentos da EA.

Entre os aspectos preponderantes nas respostas dos ACS destaca-se a pouca disponibilidade de informação sobre EA, bem como a dificuldade de multiplicação de tal conhecimento para a comunidade, o que impacta negativamente no seu papel como educador ambiental e de saúde. Torna-se evidente a necessidade de aprimoramento das estratégias de educação permanente, as quais podem auxiliar o preenchimento de lacunas relativas às competências desejáveis dos ACS, a fim de garantir, de fato, a prevenção e promoção da população em foco (BRASIL, 2003).

\section{Existência de algum trabalho de preservação ambiental na comunidade}

Os ACS não souberam informar sobre trabalhos desenvolvidos na área da UBSF que visassem à preservação ambiental.

\section{Finalidade da preservação ambiental}

Foram selecionadas quatro temáticas, solicitando-se aos entrevistados para pontuar aquela capaz de justificar a necessidade de preservação ambiental, a partir da pergunta: "Você acha que a preservação do meio ambiente é importante (assinale apenas uma alternativa)”. A maioria das respostas (temática IV) dos ACS vai ao encontro da corrente de pensamento conservacionista (CARDOSO, 2009), pois ao prevenir e promover hábitos e ambientes saudáveis os ACS estão buscando um caminho para a sustentabilidade. Alguns ACS também tiveram outra visão situada, do ponto da vista da ecologia, no preservacionismo, uma vez que tentam salvaguardar o ambiente das ações antrópicas (CARDOSO, 2009).

\section{Conhecimento sobre EA}

Ao se analisar o entendimento dos entrevistados acerca da EA, pôde-se identificar duas categorias.

Através da análise dos discursos expressos pelos ACS é possível inferir que a concepção de EA está aquém do conceito mais abrangente e complexo que a temática 
assume no contexto da práxis. Existe uma explícita ligação do meio ambiente com a ideia de "limpeza", uma identificação que provavelmente representa um resquício do higienismo sanitário difundido no inicio do século XX (JUNIOR GÓIS, 2003; PENTEADO et al., 2005).

No entanto, ao comparar as falas dos entrevistados acerca da relação do ambiente com o processo de adoecimento e da importância da EA, percebe-se que a definição expressa de EA é mais pobre do que a incorporação da mesma na prática profissional. Ou seja, há um lapso entre o saber e o fazer, o que permite inferir que os conhecimentos dos ACS acerca da EA, como instrumento de PS, foram assimilados de modo incipiente - nos cursos destinados à formação ou se originam, de algum modo, do senso comum (IANNI \& QUITÉRIO, 2009).

\section{Educação Ambiental como função dos ACS}

Com a finalidade de verificar se os entrevistados entendem a EA como incumbência do ACS, foi utilizada a pergunta: "Você considera a Educação Ambiental como uma de suas funções como ACS?". Vinte dos 21 entrevistados consideram a EA como essencial para a sua prática profissional.

\section{CONSIDERAÇÕES FINAIS}

A EA tem relevância no processo de formação dos profissionais de saúde (SIQUEIRA-BATISTA et al., 2009), por possuir, em seu bojo, elementos que, por si só, se constituem em poderosos agentes transformadores. Cabe destacar, no âmbito da ESF, o papel dos ACS, os quais, através do vínculo e da corresponsabilidade com a comunidade (GOMES et al., 2009), podem auxiliar, decisivamente, na redução dos impactos ambientais e no controle social referente ao uso racional dos recursos naturais.

A partir desta perspectiva, deve-se procurar discutir o impacto ecológico das ações cotidianas com os profissionais da saúde - tendo em vista sua formação técnica, mas, sobretudo, sua participação na sociedade como cidadãos - o que nem sempre é fácil. De fato, em uma pesquisa semelhante ao presente trabalho, realizada por Ianni e Quitério (2009), em quatro municípios da região metropolitana de São Paulo, foram pontuados os seguintes entraves quanto à incorporação da EA ao contexto da promoção à saúde: (1) a inconsistência com que esses temas são abordados nos documentos normativos; (2) a quase inexistência de instrumentos concretos para o enfrentamento desta problemática; (3) a complexidade das questões ambientais e sua não contemplação 
nos instrumentos normativos; e (4) a discordância entre a capacidade de enxergar e agir sobre um problema ambiental identificado (IANNI \& QUITÉRIO, 2009).

Por tudo isto, reconhece-se que as equipes de ESF estão longe de cumprir plenamente o seu papel, no que tange às questões ambientais. Assim, pois, de um ponto de vista operacional, cabe às equipes de saúde da família - e, especialmente, aos ACS organizar e dar voz à população, em especial aquelas parcelas marginalizadas, monitorando as ações de educação em saúde e ambiente, em parceria com a comunidade, trabalhando intersetorialmente com associações de bairros, igrejas, esferas públicas e privadas (MONKEN et al., 2008) em prol do bem comum, de uma mais adequada qualidade de vida dos cidadãos. Entretanto, estas não devem ser responsáveis por sanar todas as mazelas do território onde atuam, participando, outrossim, como catalisadoras do processo de mudança no tratamento das questões ambientais, auxiliando na ação da comunidade para tomada de decisões. Com efeito, mais que uma ação de proteção à saúde, ao poupar internações e óbitos por doenças “evitáveis”, a EA pode ser vista como um poderoso motriz nos processos de promoção da saúde, condição essencial para a construção de uma sociedade justa e harmônica nas suas relações com o meio ambiente.

\section{REFERÊNCIAS}

AZEVEDO, E.A. Exclusão sanitária em Belo Horizonte - MG: caracterização e associação com indicadores de saúde [Tese]. Belo Horizonte (MG): Universidade Federal de Minas Gerais, 2003.

BARDIN, L. Análise de conteúdo. Lisboa: Persona, 1977.

BRASIL. Conferência das Nações Unidas sobre Meio Ambiente e Desenvolvimento. 3.ed. Brasilia: Senado Federal, Subsecretaria de Edições Técnicas, 2001.

BRASIL/DAB. DEPARTAMENTO DE ATENÇÃO BÁSICA. Atenção Básica e a Saúde da Família - diretriz conceitual [internet]. Ministério da Saúde. Disponível em $>$ http://dab.saude.gov.br/atencaobasica.php<. Acesso em 03 de janeiro de 2013.

BRONBERGER, S.M. A estratégia saúde da família numa perspectiva ambiental para a promoção da saúde. Boletim da Saúde. 2003;17(2).

CAMPOS, G.W.S. Papel da Atenção Básica em Saúde na formação médica Diretrizes. Cadernos ABEM. 2007; 3:6-10.

CAPRA, F. O ponto de mutação: a ciência, a sociedade e a cultura emergente. São Paulo: Cultrix, 2006. 
CARDOSO, C.A.F.; JUNQUEIRA, T.; COSTA, L.O.; GOMES, A.P.; MATTOS, E.A.; SIQUEIRA-BATISTA, R. Conversações entre Charles Darwin e Carlos Chagas: a infecção por Trypanosoma cruzi sob uma perspectiva ecoevolutiva e pedagógica. Cadernos Saúde Coletiva. 2009;17(4): 811-825.

DUNCAN, B.B.; SCHMIDT, M.I.; GIUGLIANI, E.R.J. Medicina ambulatorial. Condutas de Atenção Primária Baseadas em Evidências. Porto Alegre: Artmed; 2006.

GOMES, A.P.; SANTOS, M.A.; RÔÇAS, G.; GONÇALVES, M.L.C.; SIQUEIRABATISTA, R. Vida e morte na Amazônia: o desmatamento e a ecoepidemiologia da infecção por Trypanosoma cruzi como temática para a educação ambiental na formação de profissionais de saúde. Caderno de Resumos do Encontro Nacional de Ensino de Ciências da Saúde e do Ambiente. Niterói: UNIPLI 2008;1:440-446.

GOMES, K.O.; COTTA, R.M.M.; CERCHIGLIA, M.L.; MITRE, S.M.; SIQUEIRABATISTA, R.A. A práxis do agente comunitário de saúde no contexto do programa saúde da família: reflexões estratégicas. Saúde e Sociedade. 2009;18(4):744-755.

GUIMARÃES, M. Educação ambiental: no consenso um embate? Campinas: Papirus, 2000 .

HELLER, L. Saneamento e saúde. Brasília: OPAS/OMS; 1997.

HIPÓCRATES. Conhecer, Cuidar, Amar. Seleção de textos e apresentação de Jean Salem. São Paulo: Landy, 2002.

IANNI, A.M.Z.; QUITÉRIO, L.A.D. A questão ambiental urbana na atenção básica e o PSF. In: COHN, A. Saúde da família e SUS: Convergências e dissonâncias. Rio de Janeiro: Beco do Azougue, 2009.

IBGE. Instituto Brasileiro de Geografia Estatística. Demografia social. Disponível em > http://www.ibge.gov.br/home/<. Acesso em 01 de janeiro de 2013.

JUNIOR GÓIS, E. Higienismo e Positivismo no Brasil: unidos e separados nas campanhas sanitárias (1900-1930). Dialogia. 2003;2:21-32.

LOUREIRA, C.F.B. Educação ambiental transformadora. Ministério do Meio Ambiente. Identidades da educação ambiental brasileira. Brasília: Ministério da Saúde; 2004. p.65-84.

MINAYO, M.C.S. O desafio do conhecimento - pesquisa qualitativa em saúde. 9 ed. São Paulo/Rio de Janeiro: HUCITEC/ABRASCO, 2006.

MONKEN, M.; PEITER, P.; BARCELLOS, C.O. Território na Saúde: construindo referências para análises em saúde e ambiente. In: MIRANDA, A.C.; BARCELLOS, C.; MOREIRA, J.C.; MONKEN, M. Território, Ambiente e Saúde. Rio de Janeiro: Fiocruz, 2008, p. 23-41.

PAULA, J.A. Fundamentos históricos e metodológicos da questão ambiental. In: PAULA, J.A.; BARBIERI, A.F. Biodiversidade, população e economia: uma região de mata atlântica. Belo Horizonte: UFMG/CEDEPLAR; 1997, p.201-256.

PENTEADO, R.Z.; CHUN, R.Y.S.; SILVA, R.C. Do higienismo às ações promotoras de saúde: a trajetória em saúde vocal. Distúrbios da Comunicação. 2005;17(1):9-17. 
SAVASTANO, H. Abordagem do binômio saúde-doença e do conceito de personalidade no ecossistema: implicações em saúde pública. Revista de Saúde Pública. 1980:14(1):137-142.

SECRETARIA DE GESTÃO DO TRABALHO E EDUCAÇÃO NA SAÚDE, Departamento de Gestão da Educação na Saúde. Perfil de competências profissionais dos agentes comunitários de saúde. Brasília: Ministério da Saúde, 2003.

SIQUEIRA-BATISTA, R. Deuses e homens. Mito, filosofia e medicina na Grécia antiga. São Paulo: Landy, 2003.

SIQUEIRA-BATISTA, R.; RÔÇAS, G.; GOMES, A.P.; ALBUQUERQUE, V.S.; ARAÚJO, F.M.B.; MESSEDER, J.C. Ecologia na formação dos profissionais de saúde: promoção do exercício da cidadania e reflexão crítica comprometida com a existência. Revista Brasileira de Educação Médica. 2009;33(2):271-275.

SIQUEIRA-BATISTA, R.; GOMES, A. P.; RÔÇAS, G.; COTTA, R. M. M.; RUBIÃO, E. C. N.; PISSINATTI, A. Moléstia de Chagas e ecologia profunda: a "luta antivetorial" em questão. Ciência e Saúde Coletiva, v. 16, n. 1, p. 677-687, 2011.

SRINIVASAN, S.; O'FALLON, L.R.; DEARRY, A. Creating healthy communities, healthy homes, healthy people: initiating a research agenda on the built environment and public health. American Journal of Public Health. 2003;93(9):1446-1450.

TEIXEIRA, J.C.; PUNGIRIM, M.E.M.C. Análise da associação entre saneamento e saúde nos países da América Latina e do Caribe, empregando dados secundários do banco de dados da Organização Pan-Americana de Saúde - OPAS. Revista Brasileira de Epidemiologia. 2005;8(4):365-376.

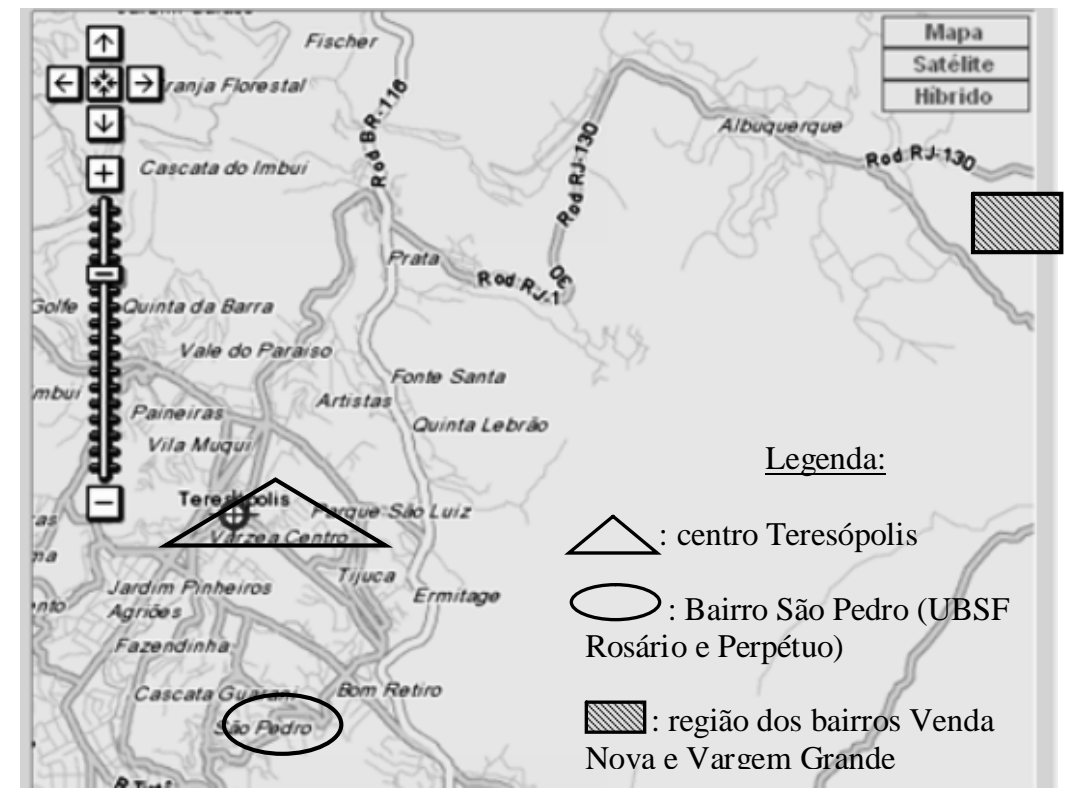

Figura 1. Unidades Básicas de Saúde da Família estudadas. Adaptado de www.telelistas.net. 
Quadro 1. Perguntas do roteiro de entrevista aplicado aos agentes comunitários de saúde.

\begin{tabular}{|c|l|}
\hline Número & \multicolumn{1}{c|}{ Questão } \\
\hline $\mathbf{1}$ & O que faz as pessoas de sua comunidade adoecerem? \\
\hline $\mathbf{2}$ & $\begin{array}{l}\text { Você acredita que o ambiente em que as pessoas vivem pode levar ao aparecimento } \\
\text { de doenças? Dê exemplo(s) dessa correlação. }\end{array}$ \\
\hline $\mathbf{3}$ & $\begin{array}{l}\text { Você já recebeu capacitação sobre meio ambiente ou educação ambiental? Você } \\
\text { acha importante? }\end{array}$ \\
\hline $\mathbf{4}$ & $\begin{array}{l}\text { Você conhece algum trabalho de preservação ambiental na comunidade? } \\
\mathbf{5}\end{array}$ \\
$\begin{array}{l}\text { Você acha que a preservação do meio ambiente é importante (assinale apenas uma } \\
\text { alternativa): } \\
\text { I - Para que os seres humanos do futuro possam desfrutar da natureza } \\
\text { II - Para preservar a natureza e os animais } \\
\text { III - Para o bem-estar do homem } \\
\text { IV - Para a saúde do planeta }\end{array}$ \\
\hline
\end{tabular}

